\title{
A morphometric survey of the parasellar region in more than 2700 skulls: emphasis on the middle clinoid process variants and implications in endoscopic and microsurgical approaches
}

\author{
Akshay Sharma, BA, ${ }^{1}$ Gabrielle E. Rieth, MS, ${ }^{1}$ Joseph E. Tanenbaum, BA, ${ }^{1,2}$ \\ James S. Williams, MD, ${ }^{3}$ Nakao Ota, MD, ${ }^{4}$ Srikant Chakravarthi, MD, ${ }^{5}$ Sunil Manjila, MD, ${ }^{6}$ \\ Amin Kassam, MD, ${ }^{5}$ and Bulent Yapicilar, MD ${ }^{1,7}$ \\ ${ }^{1}$ Case Western Reserve University School of Medicine; ${ }^{2}$ Department of Epidemiology and Biostatistics, Case Western Reserve \\ University; and Departments of ${ }^{3}$ Radiology and ${ }^{7}$ Neurosurgery, MetroHealth Medical Center, Cleveland, Ohio; ${ }^{4}$ Department \\ of Neurosurgery, Teishinkai Hospital, Sapporo, Hokkaido, Japan; ${ }^{5}$ Aurora Neurosciences Center, Milwaukee, Wisconsin; and \\ ${ }^{6}$ Department of Neurosciences, McLaren Bay Neurosurgery Associates, Bay City, Michigan
}

\begin{abstract}
OBJECTIVE The middle clinoid process (MCP) is a bony projection that extends from the sphenoid bone near the lateral margin of the sella turcica. The varied prevalence and morphological features of the MCP in populations stratified by age, race, and sex are unknown; however, the knowledge of its anatomy and preoperative recognition on CT scans can aid greatly in complication avoidance and management. The aim of this study was to further illustrate the surgical anatomy of the parasellar region and to quantify the incidence of MCP and caroticoclinoid rings (CCRs) in dried preserved human anatomical specimens.
\end{abstract}

METHODS The presence, dimensions, morphological classification (incomplete, contact, and CCR), and intracranial relations of the MCP were measured in 2726 dried skull specimens at the Hamann-Todd Osteological Collection at the Cleveland Museum of Natural History. Specific morphometric data points were recorded from each of these hemiskulls, and categorized based on age, sex, and ethnicity. Linear and logistic regressions were used to determine associations between explanatory variables and MCP morphology. Computed tomography scans of the skull specimens were obtained to explore radiological landmarks for different types of MCPs. Illustrative intraoperative videos were also analyzed in the light of these crucial surgical landmarks.

RESULTS The sample included 2250 specimens from males and 476 from females. Specimens were classified as either "white" (60.5\%) or "black" (39.2\%). An MCP was found in $42 \%$ of specimens, with $60 \%$ of those specimens presenting bilaterally. Fully ossified CCR comprised $27 \%$ of all MCPs, and contact (defined as contact without ossification between MCP and anterior clinoid process) comprised $4 \%$ of all MCPs. White race (relative to black race) and increasing age were significant predictors of MCP presence $(p<0.001)$. White race was significantly associated with greater average MCP height $(p<0.001)$. Among skulls with CCR, both male sex and older age $>70$ years relative to $<50$ years) were associated with increased CCR diameter $(p<0.001)$. No other significant predictors or associations were observed. The CT scans of skulls replicated and validated the authors' morphometric observations on incomplete, contact, and CCR patterns adequately. The surgical strategies of clinoid bone removal are validated, with appropriate video illustrations.

CONCLUSIONS Variations in the patterns of bony MCPs can pose a significant risk for injury to the internal carotid artery during parasellar procedures, especially those involving clinoidectomy and optic strut drilling. Understanding parasellar anatomy, especially on skull-base CT imaging, may be integral to surgical planning and preoperative risk counseling in both transcranial and extended endonasal procedures, as well as to preparing for complications management perioperatively.

https://thejns.org/doi/abs/10.3171/2017.2.JNS162114

KEY WORDS skull base; clinoid process; endoscopic endonasal approach; middle clinoid process; internal carotid artery; sphenoid sinus; oncology

ABBREVIATIONS ACP = anterior clinoid process; CCR = caroticoclinoid ring; CTA = CT angiography; EEA = expanded endonasal approach; ICA = internal carotid artery; ICB = interclinoid bridge; LBW = lateral bone window; LTC = lateral tubercular crest; $M C P=$ middle clinoid process; $M O C R=$ medial opticocarotid recess; OCE = opticocarotid elevation; $\mathrm{OS}=$ optic strut; $\mathrm{PCP}=$ posterior clinoid process; $\mathrm{TS}=$ tuberculum sellae.

SUBMITTED August 14, 2016. ACCEPTED February 2, 2017.

INCLUDE WHEN CITING Published online August 11, 2017; DOI: 10.3171/2017.2.JNS162114. 
$\mathrm{T}$ HE central skull base can be surgically approached via transcranial microsurgery as well as expanded endonasal approaches (EEAs), especially in the case of parasellar tumors. ${ }^{18-20,22,34}$ The middle clinoid process (MCP) is a variable, small projection of bone from the sphenoid bone of the skull near the anterolateral margin of the sella turcica, exhibiting different morphological patterns crucial to surgical approaches, whether these are microsurgical or endonasal endoscopic. During endoscopic endonasal approaches to the pituitary gland, the MCP can serve as a landmark to locate the anteromedial roof of the cavernous sinus, as well as a marker of the transition between the cavernous and clinoid segments of the internal carotid artery (ICA). ${ }^{12}$ To prevent injuries of the ICA, an understanding of the location of the MCP is necessary, because it is a bony protrusion that fixes the artery medially. ${ }^{38}$ During transcranial microsurgical approaches, the MCP is a landmark for cavernous sinus surgery as well as tumor resections, typically in meningiomas involving the caroticoclinoid ligament. $^{12}$

The MCP, however, is variably present in human specimens surveyed, and when present, it varies greatly in size. Furthermore, extensive ossification of the caroticoclinoid ligament, by which the MCP is attached to the anterior clinoid process (ACP), can result in the formation of a bony caroticoclinoid ring $(\mathrm{CCR})$ surrounding the ICA., ${ }^{1,30}$ Presence of the bony CCR (often called a foramen instead of a ring) adds further complexity to both endonasal and transcranial tumor resections, such as for meningiomas and pituitary adenomas. To mobilize the ICA while avoiding subsequent damage, the CCR must be dissected without splintering. We have observed that the relation of the ICA to partial or fully formed CCRs varies from complete encircling of the ICA to transposition or displacement of the artery. This holds high clinical significance, especially when treating parasellar meningiomas and ICA aneurysms in that region.

Given the clinical significance of the MCP in both transcranial microsurgical and endoscopic endonasal procedures, the objective of this study is to further illustrate the surgical anatomy of the parasellar region, with particular focus on the MCP, and to quantify the incidence of MCP and CCR in dried, preserved human anatomical specimens. This study also aims to delineate the osseous and intracranial neurovascular relationships of each pat- tern with reconstructed images from thin-cut head CT scans, and to illustrate the utility of recognizing these bony variations during endoscopic endonasal as well as transcranial microsurgical procedures.

\section{Methods}

The Hamann-Todd Osteological Collection at the Cleveland Museum of Natural History represents one of the largest and most diverse documented human skeletal collections in the world. ${ }^{23,27}$ Data consisting of the stated age, sex, race, and relevant clinical information for each evaluated specimen were recorded.

A total of 2892 cranial specimens were examined along existing sagittal, coronal, and axial hemisections to provide access to 5784 parasellar areas for morphometric assessment of the endocranial surface of the skull base (Fig. 1). Skulls with significant damage to the parasellar region that prohibited accurate measurement were excluded $(n=166)$.

The presence, dimensions, and intracranial relations of the MCP were measured and recorded. The MCP was classified as clinically irrelevant (height or base $<1.5$ $\mathrm{mm}){ }^{31}$ incomplete; contact (MCP almost touching the prolongation of the ACP, forming a "pseudo-ring"); or as a CCR. ${ }^{23}$ The height of MCPs classified as incomplete and contact was measured from the origin of the base on the parasellar wall to the most lateral extension of the process, whereas the diameter of the ovular base for all classes was measured at its greatest length in an oblique horizontal plane, closer to the midline superiorly than inferiorly. ${ }^{31}$ For incomplete MCPs, the distance between the most lateral aspect of the MCP and the most medial aspect of the ACP was measured. The widest diameter of the foramen formed by contact clinoid processes and CCRs was also obtained. Distances from the opticocarotid elevation (OCE) and the optic strut (OS) were taken from the superior aspect of the base of the MCP. The widest diameters of the proximal and distal foramen of the bony carotid canal, and the width of the OS extending from the posterolateral vertex of the optic canal to the posteromedial border of the OS was recorded for each specimen in which a clinically relevant MCP was identified. Pictorial summaries of measurements and morphological classifications are included in Figs. 2 and 3.
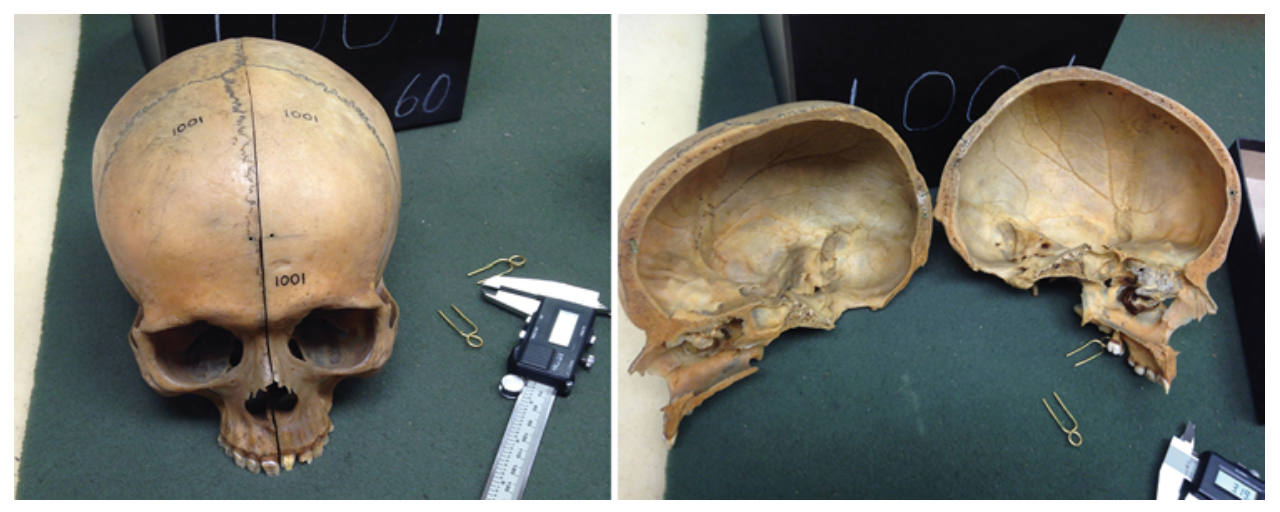

FIG. 1. Full (left) and hemisected (right) skull specimens from the Hamann-Todd Collection at the Cleveland Natural History Museum. Figure is available in color online only. 

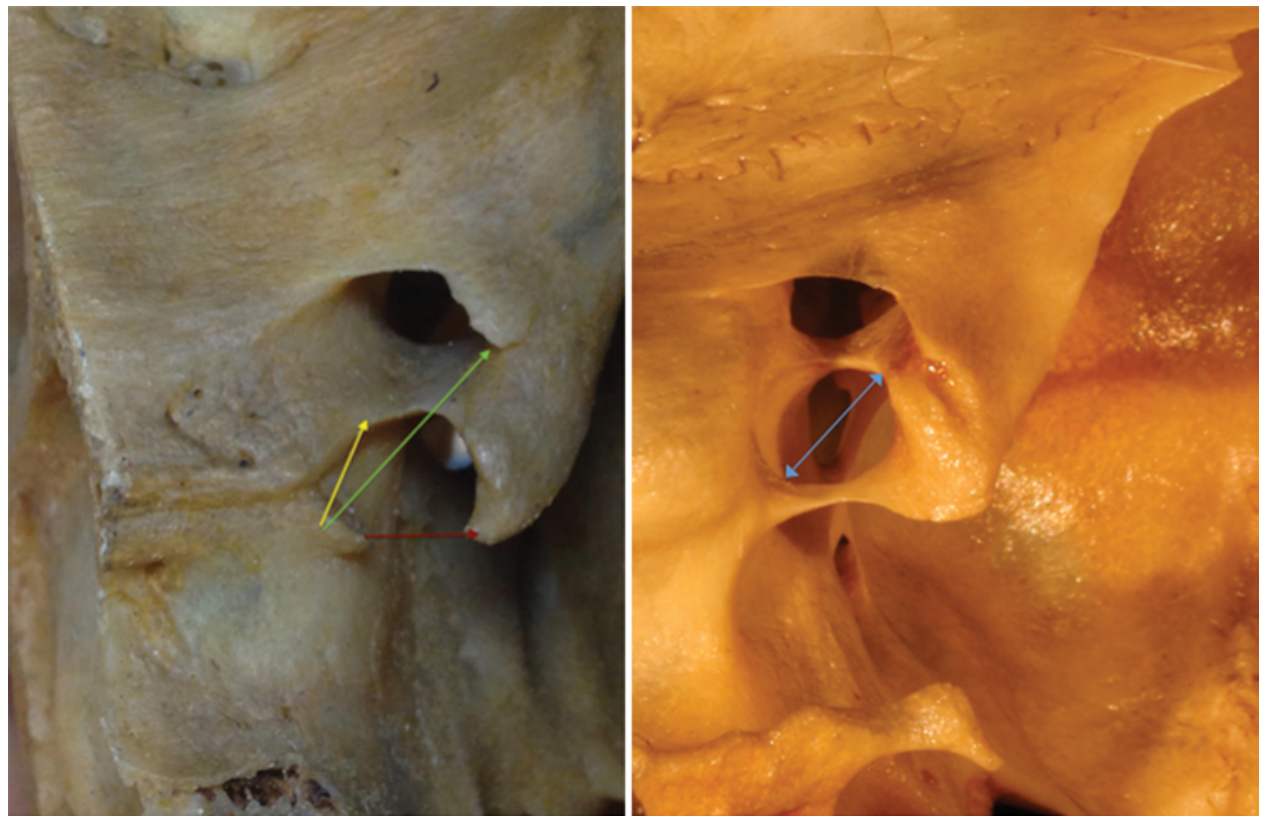

FIG. 2. Skull specimens showing morphometric parameters including the diameter of the base and the height of the process, along with the anatomical bony boundaries. Left: The length from the lateral aspect of the MCP to the medial aspect of the ACP (horizontal red arrow), distance from the base of the MCP in the lateral edge of the OS (long green arrow), and the distance from the base of the MCP to the origin of the OCE (upward-pointing, yellow arrow). Right: Widest diameter of the caroticoclinoid foramen was measured in contact and complete morphologies (blue arrow). Figure is available in color online only.

A digital caliper (Mitutoyo America Corp.) was used to obtain all intracranial measurements. Interobserver reliability testing was performed by 2 of the authors (A.S. and G.R.), who performed blinded measurements using the method described above within a 2-day period. Interobserver and intraobserver reliability for all measurements was assessed with the intraclass correlation coefficient over a sample of 150 specimens $\left(\rho^{*}>0.75\right) .{ }^{37}$ The unpaired Student t-test and Fisher's exact test were used to examine specific differences. A p value of $<0.05$ was used as a threshold for significance. SPSS Statistics 22.0 (IBM, Inc.) was used for all data analysis.

Data on MCP base diameter and MCP height were assessed for normality. The MCP base diameter data were normally distributed; however, the MCP height was skewed. We log-transformed the MCP height data to obtain normally distributed data. Logistic regression was used to determine predictors of MCP presence and laterality, presence of contact, and presence of a fully formed caroticoclinoid foramen with age, sex, and race as covariates. Among specimens with MCP, ordinary least squares linear regression was used to determine the association between MCP base diameter and age, sex, and race. We built an identical model using log-transformed MCP height as our dependent variable.

The CT scanning was performed on a Philips 256-slice iCT scanner on 23 specimens chosen from the subsets of the varied morphological categories of the MCP. The skulls were positioned in a manner that would have been consistent with the body being in the supine position, placed in a foam headholder. A standard scout image was taken and imaging was performed from below the sella turcica through the entirety of the petrous apices. Scan- ner collimation was set at $0.625 \mathrm{~mm}$, with an image slice thickness of $0.9 \mathrm{~mm}$. The reconstruction interval was 0.45 $\mathrm{mm}$. Scan settings were as follows: $\mathrm{mA} 350(258 \mathrm{~mA} \times$ 0.75 seconds), $\mathrm{kVp} 120$, rotation time 0.75 , and pitch 0.552 . The reconstruction algorithm was Philips ultra-high-resolution "Y-sharp (YE filter)." Isotropic data were used to create 1-mm-thick MPR reformats in the sagittal, coronal, and axial planes. Localizing measurements recorded on 3D volume-rendered images created with OsiriX MD 8.0.2 (Pixmeo) were used to validate measurements taken by hand. Interobserver and intraobserver reliability for all measurements was assessed with the intraclass correlation coefficient over a sample of 150 specimens $\left(\rho^{*}>0.75\right) .{ }^{37}$

\section{Results}

A total of 2726 cranial specimens, including 2250 (83\%) from male and $476(17 \%)$ from female donors, with complete sella turcica were examined for measurement in this study. The mean age of the sample was 48.6 years (range 18-105 years), and 60.5\% of specimens were racially identified as "white," $39.2 \%$ as "black," and < $1 \%$ were identified as "Asian" or "American Indian" (Table 1).

A clinically relevant (height or base $>1.5 \mathrm{~mm}$ ) MCP was found along the medial border of the carotid sulcus in $42 \%$ of specimens (1146 of 2726), with $60 \%$ of these (682 of 1146) presenting with bilateral processes (Table 1). In unilateral specimens, the MCP was found on the right in $57 \%$ of skulls (264 of 464) and on the left in $43 \%$ (200 of 464) ( $\mathrm{p}<0.0001)$. Of the $1854 \mathrm{MCPs}$ identified, $69 \%$ were classified as incomplete (1275 of 1854), $4 \%$ as contact ( 75 of 1854), and $27 \%$ were identified as fully formed caroticoclinoid foramen (504 of 1854) (Table 2). 


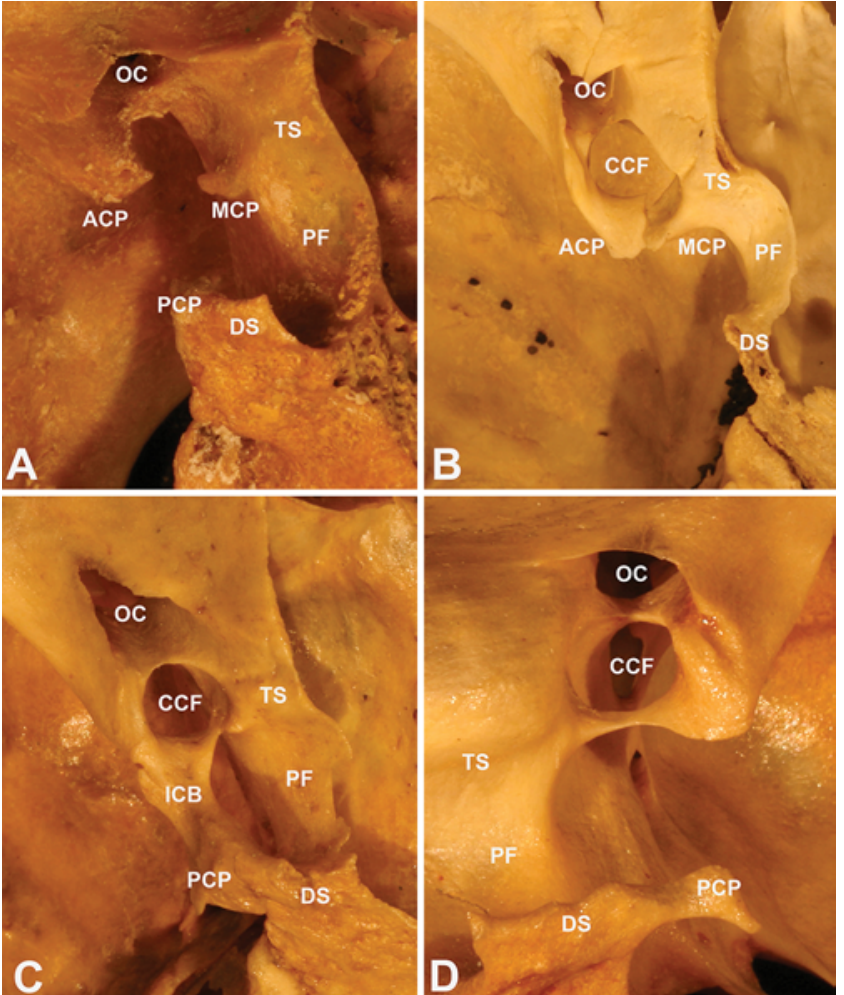

FIG. 3. The MCP was found along the medial aspect of the carotid sulcus oriented immediately posterolaterally, extending superolaterally toward the ACP. The following bony morphologies of MCPs were observed: incomplete ossification on the left side viewed from directly superior $(\mathbf{A})$; contact on the left side in a superior right oblique view (B); the CCR with concurrent ICB on the left side viewed in a direct superior view (C); and a sole CCR on the right viewed from directly superior (D). CCF = caroticoclinoid foramen; $D S=$ dorsum sellae; OC = optic canal; $\mathrm{PF}=$ pituitary fossa. Figure is available in color online only.

The mean distance between the most lateral aspect of the incomplete MCP and the most medial aspect of the ACP measured $3.80 \mathrm{~mm}$. The average size of an incomplete MCP was $2.59 \mathrm{~mm}$ in height and $4.16 \mathrm{~mm}$ in diameter at its base. Processes exhibiting a contact morphology were found to be $3.65 \mathrm{~mm}$ in height, with a $4.48-\mathrm{mm}$ base.

TABLE 1. Demographic variation and incidence of the MCP in a sample of 2726 skulls

\begin{tabular}{cc}
\hline \multicolumn{1}{c}{ Variable } & Value $^{*}$ \\
\hline Male & $2250(83)$ \\
\hline Age $(\mathrm{yrs})$ & $48.6 \pm 15.6$ \\
\hline Race & \\
\hline White & $1648(60.5)$ \\
\hline Black & $1068(39.2)$ \\
\hline Other & $10(0.3)$ \\
\hline Specimens w/ MCP & $1146(42)$ \\
\hline Bilat & $682(60)$ \\
\hline Total MCP & 1854 \\
\hline
\end{tabular}

* Values are expressed as the mean \pm SD for continuous variables and number (\%) for categorical variables.
TABLE 2. Morphological classifications of 1854 identified MCPs

\begin{tabular}{lc}
\hline Classification & Prevalence* $^{*}$ \\
\hline Incomplete & $1275(69)$ \\
\hline Contact & $75(4)$ \\
\hline CCR & $504(27)$ \\
\hline
\end{tabular}

* Values are expressed as number (\%) for categorical variables.

The mean diameter of the "pseudo-ring" of the contact MCP was $5.63 \mathrm{~mm}$. Caroticoclinoid foramina were found to have a mean base diameter of $5.03 \mathrm{~mm}$, with a mean ring diameter of $5.52 \mathrm{~mm}$. The mean difference between the average diameters of the base of the incomplete processes as compared with those of the ring-forming morphologies was calculated to be $0.87 \mathrm{~mm}$, and was statistically significant $(\mathrm{p}<0.0001)$.

Regardless of classification, 99\% (1831 of 1854) of all MCPs were located within the anterior one-third of the lateral bone window (LBW) of the sella turcica, the region defined by the line between the ACP and the posterior clinoid process $(\mathrm{PCP})$, the medial edge of the carotid sulcus, the posterior border of the OS, and the lateral border of dorsum sellae. ${ }^{40}$ The remaining $1 \%$ were found in the middle one-third of the LBW. The MCP was found along the medial aspect of the carotid sulcus, just inferior to the lateral tubercular crest (LTC), oriented posterolaterally, eventually curving superiorly toward the ACP (Fig. 4). The superior aspect of the base of the MCP was found $6.25 \mathrm{~mm}$ posteromedially from the posterior border of the OS, $3.70 \mathrm{~mm}$ inferior to the OCE. The width of the OS, taken between the most lateral and medial borders, was $4.09 \mathrm{~mm}$. Intraclass correlation coefficients between measurements taken by hand and those taken on CT scans for the 23 imaged specimens were all $\rho>0.85$. Figure 5 depicts the utility of $\mathrm{CT}$ imaging in recreating the bony anatomy of the region.

An interclinoid bridge (ICB), an osseous bar connecting the ACP and the $\mathrm{PCP},{ }^{40}$ was found in $4 \%$ of all parasellar regions examined (224 of 5318). The incidence of the ICB among specimens with MCP present was significantly greater (7.8\%) when compared with the incidence of the ICB in specimens in which the MCP was absent $(2.3 \%)$ (p $<0.0001)$. Furthermore, when stratified by ring-forming and incomplete morphology, specimens with a contact or CCR had a significantly greater incidence of ICBs (19.7\%) as compared with the incidence in specimens with incomplete MCPs $(2.5 \%)(\mathrm{p}<0.0001)$.

When stratified by sex, there was no significant difference $(p=0.6841)$ in the presence of an MCP. An MCP was identified in $44 \%$ of biologically male skulls (990 of 2249), and a clinically relevant MCP was identified in $43 \%$ of biologically female skulls (204 of 475). Bilateral MCPs were identified in 560 of 966 male specimens (57.97\%), and in 122 of 291 female specimens $(41.92 \%)(\mathrm{p}=0.1473)$. Male sex was significantly associated with a larger ring diameter in ring-forming MCP variations $(\beta=0.85,95 \%$ CI 0.39-1.31; $\mathrm{p}<0.001)$.

Specimens from white donors had significantly greater odds of having an incomplete MCP relative to skulls from black donors (OR 2.29, 95\% CI 1.93-2.71). When stratified 


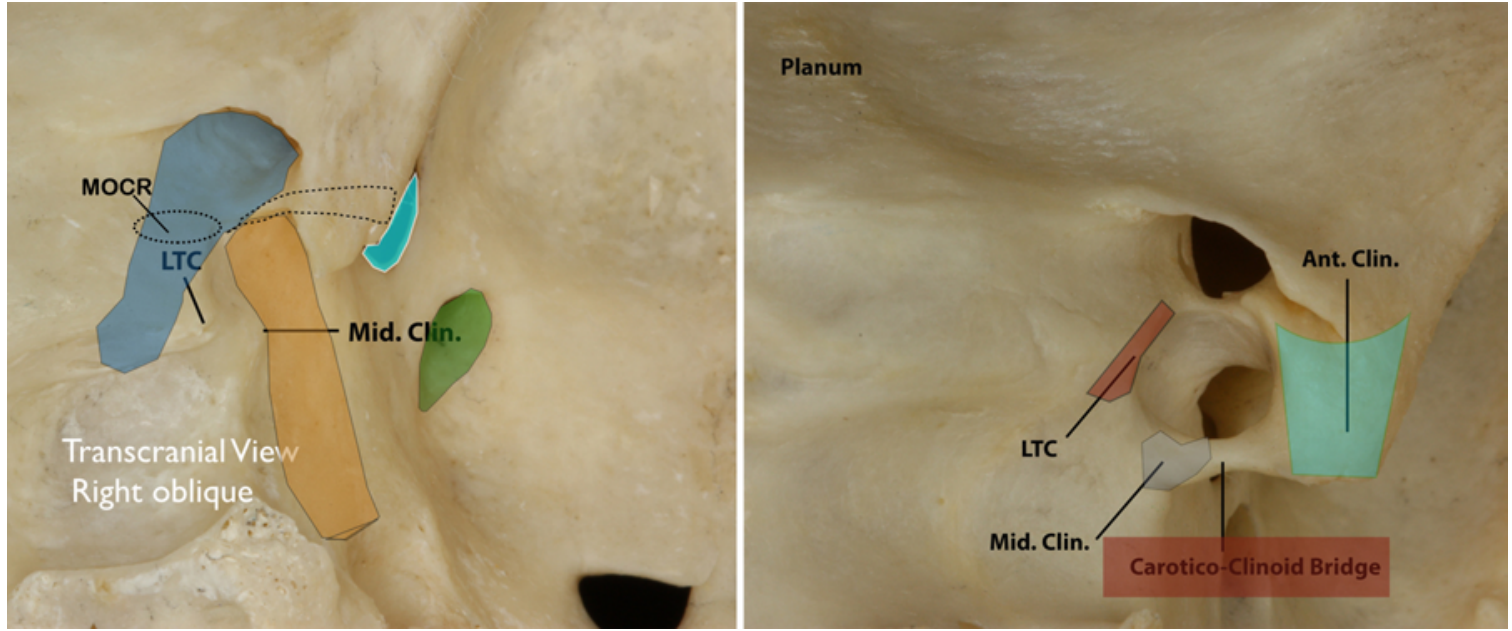

FIG. 4. Comparative osseous landmarks delineating the location and extent of the right MCP seen transcranially. Left: A right oblique view of an incomplete-type MCP. The dotted wedge represents the OS; the adjacent turquoise area indicates the superior orbital fissure; and the green area below marks the foramen rotundum. Right: A superolateral view of a CCR. The LTC is seen as a ridge situated at the superomedial aspect of the carotid sulcus here, corresponding to the lateral tubercular recess in the transsphenoidal view. The MOCR is also demonstrated here as a teardrop-shaped osseous indentation formed at the medial junction of the optic canal and the paraclinoid carotid canal. Dorsally, this is represented by a teardrop-shaped area bounded by the inferior aspect of the LTC and the medial aspect of the junction of the posterior and superior surfaces of the OS, as well as the lateral aspect of the tuberculum. Of note, the MCP is always seen inferior to the LTC and the distal osseous arch of the carotid sulcus connects the lateral opticocarotid recess to the lateral tubercular recess. Ant. Clin., Mid. Clin. $=$ anterior clinoid, middle clinoid. Figure is available in color online only.

by race (Table 3), a clinically relevant MCP was identified in 52\% of parasellar regions obtained from individuals documented as white (866 of 1648), compared with $30 \%$ in parasellar regions from individuals documented as black (325 of 1068) ( $p<0.0001)$. In both black and white specimens, the MCP presented bilaterally in 57\% of cases. In white specimens specifically, $73 \%$ of identified MCPs were classified as incomplete, $4 \%$ as contact, and $23 \%$ as caroticoclinoid foramen. In black specimens, $57 \%$ of identified MCPs were classified as incomplete, $4 \%$ as contact, and $39 \%$ as caroticoclinoid foramen. Relative to black specimens, a significantly greater average MCP height was measured in white specimens $(\beta=1.11,95 \%$ CI 1.04-1.19; $\mathrm{p}<0.001)$.

Age older than 50 years was also a significant predictor of incomplete MCP presence relative to age 50 years or younger (OR 1.42, 95\% CI 1.20-1.67; p < 0.001). Age older that 70 years was significantly associated with larger

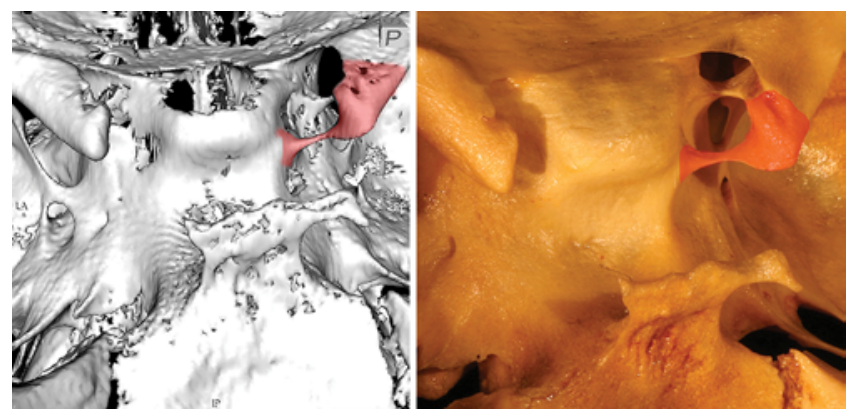

FIG. 5. A CT scan of the head with volumetric construction depicting the location and morphology of the MCP (left), with accurate visualization and correlation to direct cadaveric measurement (right). Figure is available in color online only.
CCR basal diameter $(\beta=0.83,95 \%$ CI 0.81-1.6; $\mathrm{p}<0.05)$. We found no significant predictors of caroticoclinoid foramen or presence of contact among specimens with MCP.

Two illustrative cases of clinoidectomy, 1 treated with an endoscopic endonasal and 1 with a microsurgical approach, provided and selected by the senior surgeon-authors (A.K. and N.O.), are shown. Use of careful drilling and safe directional forces in "egg-shell" removal of the thinned-out bone allow for removal without injuring the carotid artery (Videos 1 and 2).

VIDEO 1. Clip showing removal of MCP during transcranial microsurgical approach. Courtesy of Nakao Ota, MD, Stroke Center, Department of Neurosurgery, Teishinkai Hospital, Sapporo, Japan. Copyright Akshay Sharma. Published with permission. Click here to view.

TABLE 3. Incidence and classification of the MCP in skulls from white versus black donors

\begin{tabular}{lccr}
\hline \multicolumn{1}{c}{ Variable } & White Skulls $^{*}$ & Black Skulls $^{*}$ & p Value \\
\hline Sample size & 1648 & 1068 & \\
\hline Age $(\mathrm{yrs})$ & $53 \pm 14.5$ & $41.7 \pm 14.7$ & $<0.0001$ \\
\hline Male & $1436(87)$ & $806(75)$ & $<0.0001$ \\
\hline Specimens w/ MCP & $866(52)$ & $325(30)$ & $<0.0001$ \\
\hline Bilat & $490(57)$ & $185(57)$ & 0.9477 \\
\hline Total MCP & 1343 & 505 & \\
\hline Incomplete & $983(73)$ & $290(57)$ & \\
\hline Contact & $57(4)$ & $18(4)$ & \\
\hline CCR & $303(23)$ & $197(39)$ & \\
\hline
\end{tabular}

* Values are expressed as the mean \pm SD for continuous variables and number (\%) for categorical variables.

$\dagger$ Student $\mathrm{t}$-test was used for continuous variables and Fisher exact test for categorical variables. 
TABLE 4. A comparison of studies noting the incidence of the MCP

\begin{tabular}{|c|c|c|c|c|c|}
\hline \multirow[b]{2}{*}{ Authors \& Year } & \multirow{2}{*}{$\begin{array}{c}\text { Sample } \\
\text { Size }\end{array}$} & \multicolumn{3}{|c|}{ MCP } & \multirow[b]{2}{*}{ ICB } \\
\hline & & Unilat* $^{*}$ & Bilat* $^{*}$ & Total $^{*}$ & \\
\hline Keyes, 1935 & 2187 & - & - & $601(28)$ & $98(4.5)$ \\
\hline Azeredo et al., 1988 & 270 & $6(2.2)$ & $11(4.1)$ & $17(6.3)$ & $9(3.3)$ \\
\hline Inoue et al., 1990 & 50 & $11(22)$ & $7(14)$ & $18(36)$ & $2(4)$ \\
\hline Gurun et al., 1994 & 198 & $16(8.1)$ & $11(5.6)$ & $27(14)$ & $2(1.0)$ \\
\hline Erturk et al., 2004 & 171 & $41(24)$ & $20(12)$ & $61(36)$ & $14(8.2)$ \\
\hline Fernandez-Miranda et al., 2012 & 100 & $25(25)$ & $35(35)$ & $60(60)$ & - \\
\hline Peris-Celda et al., 2013 & 66 & $6(9)$ & $7(11)$ & $13(20)$ & - \\
\hline Labib et al., 2013 & 96 & - & - & $-(44)$ & - \\
\hline Present study & 2726 & $464(17)$ & $682(25)$ & $1146(42)$ & $112(4.1)$ \\
\hline
\end{tabular}

VIDEO 2. Clip showing removal of MCP during the EEA. Courtesy of Amin B Kassam, MD, Aurora Health Hospital, Milwaukee, Wisconsin. Copyright Akshay Sharma. Published with permission. Click here to view.

\section{Discussion}

An experienced skull base surgeon can deal with a CCR while handling a medial ICA aneurysm transcranially or removing tumor tissue medial to the ICA in an EEA, but this anatomy is not always familiar to even the best trained among practicing neurosurgeons. The MCP is variable in architecture and not well visualized on preoperative CT scans, unless thin-cut coronal and/or sagittal sections are analyzed or studied specifically with reconstructions. The classic MCP is a small projection of bone from the sphenoid near the anterolateral margin of the sella turcica, covered by a layer of periosteal dura mater. It can be identified at the transition between the cavernous ICA and the paraclinoidal ICA, covering the anteromedial roof of the cavernous sinus. ${ }^{12,31}$ The MCP originates medial to the anterior genu of the cavernous ICA and just superior to the proximal dural ring,,$^{12}$ and from its wide base extends dorsolaterally around the posterior aspect of the clinoidal ICA. The process extends inside the cavernous sinus below the region where the proximal and distal dural rings fuse. ${ }^{31}$ The indentation of the MCP and adjacent lateral portions of the medial tuberculum sellae (TS) form the medial opticocarotid recess (MOCR). The prominence of the recess depends on the degree of pneumatization of the MCP and TS, and should be located at the confluence of the optic canal, the cavernous sinus, the upper sella, and the anterior cranial base. ${ }^{9}$ The ACP is formed from the medial end of the sphenoid bone and is joined to the MCP by the caroticoclinoid ligament. The extent of ossification of the caroticoclinoid ligament determines the clinical prominence of the MCP. A complete ossification of the caroticoclinoid ligament that extends between the ACP and the MCP has been defined as a CCR..$^{9,12,31}$ Previously, the MOCR, which is medial to the transition between the clinoid and supraclinoid segment of the ICA, had been misidentified as the MCP. ${ }^{6,18}$ However, the MCP lies inferior to the TS and has no direct contact with the optic nerve. ${ }^{12}$
The clinoid segment of the ICA is medial to the ACP, between the proximal and distal dural rings. ${ }^{11,12,31}$ The clinoidal ICA continues from the anterior genu of the cavernous ICA as it exits the cavernous sinus. This triangular region is known as the clinoid space and is bordered inferiorly by a thin layer of dura known as the carotidoculomotor membrane, which forms the proximal dural ring at the lower margin of the ACP and the anterolateral portion of the ICA. Superiorly, the distal dural ring forms the border of the clinoid space, continuous with the medial falciform ligament and the investing dura surrounding the superomedial aspect of the ACP. ${ }^{2}$ The 2 rings are separated anteriorly but fuse posterior to the carotid artery. After passing through the distal dural ring, the ICA is considered intradural. The ICA travels anteriorly and then bends posteriorly around the caroticoclinoid ligament, continuing as the supraclinoidal segment. ${ }^{2,9,31,35}$ The presence of a completely ossified caroticoclinoid foramen may cause significant compression of the ICA, adding increasing complexity to surgical access to the parasellar region. ${ }^{9}$ This entire region forms the anterior roof of the cavernous sinus. The posterior roof is formed by a layer of dura that links the anterior and posterior petroclinoidal ligaments and the interclinoidal ligament. The PCP partially covers the posterior roof. ${ }^{2}$

The variability in incidence of the MCP specifically has been documented in several studies (Table 4). 3,11,12,16-18,23,31 Fernandez-Miranda et al. reviewed 100 fine-cut CT angiograms from their patients and, in a study of MCP incidence and prominence, reported that the MCP was identifiable in $60 \%$ of patients, and of these, $20 \%$ had an identifiable CCR. ${ }^{12}$ In the above-mentioned study, the authors found that the MCP was best visualized through thin axial-cut images at the level of the upper parasellar region. In an evaluation of 66 adult dry skulls, Peris-Celda et al. found a clinically prominent MCP present in $20 \%$ of specimens and the presence of a CCR in $3 \%$ of the specimens. ${ }^{31} \mathrm{In}$ a previous study (1935) of the Hamann-Todd collection, Keyes identified the presence of an MCP, regardless of clinical relevance, in $34.84 \%$ of 2187 skull specimens, with a fully formed caroticoclinoid foramen representing $20.3 \%$ of all MCPs found. ${ }^{23}$ In the 2726 dry specimens 
included in this study, we identified a clinically relevant (base or height $>1.5 \mathrm{~mm}$ ) MCP in $42 \%$ of skulls, with a majority $(69 \%)$ classified morphologically as incomplete, $4 \%$ as contact, and $27 \%$ as fully formed canals.

Assessing the preference for MCP presentation on either the right or left side, both Keyes and Lee et al. reported increased incidence of the MCP on the right side. ${ }^{23,25}$ Wang et al. showed increased incidence of MCPs on the right side $(20.75 \%)$ as compared with incidence on the left $(16.04 \%)$, in a study of 530 dry skulls. ${ }^{40}$ In a study of a uniformly Turkish population, however, Erturk et al. showed no significant difference in the incidence on either the right or left side. ${ }^{11}$ Findings from the current study add support to a preference for the right side, with 57\% of skulls with unilateral MCPs presenting as such ( $\mathrm{p}<$ $0.0001)$. Of skulls in which a clinically relevant MCP was found $(\mathrm{n}=1146), 60 \%$ presented with MCPs bilaterally. This is greater than what was found by Keyes $(23.41 \%)^{23}$ or Gurun et al. $(34.5 \%){ }^{16}$

Age older than 50 years was found to be a significant predictor for MCP, and was also significantly associated with greater basal diameter of CCRs, suggesting that formation may be related to a time-dependent ossification of the MCP and the adjacent caroticoclinoid ligament. However, age-related ossification within the adult anterior cranial fossa is not well reported in the literature and requires further investigation. ${ }^{41}$

A slight variation in incidence between sexes has been documented in the literature, indicating a decreased frequency in women. In a study of adult skulls from East Asia and North America, Dodo and Ishida found MCPs present in $6 \%$ of male specimens and only $3.9 \%$ of female specimens when specifically looking at Japanese populations. ${ }^{10}$ In contrast to the current literature, we found no significant difference between men and women in the incidence of MCPs ( $p=0.6841)$; however, we did note that male sex was significantly associated with greater basal diameter of CCRs.

Further stratification by ethnicity in incidence of the MCP and CCRs has been documented, showing variability between Asian and Caucasian populations. 3,6,10,11,15,26,31,32,39 A study by Lee et al. showed an incidence of $15.7 \%$ in a study of Korean skulls, including both sexes. ${ }^{25}$ Varying numbers have been found for skulls obtained from different Caucasian populations, including a $23.4 \%$ incidence in Sardinian individuals, ${ }^{26} 6.27 \%$ in Portuguese individuals, ${ }^{3}$ $14 \%$ in German individuals, ${ }^{33}$ and $34.84 \%$ in individuals identified as Caucasian Americans. ${ }^{23}$ Keyes noted that the incidence of MCPs was more than $15 \%$ greater in white individuals when compared with black individuals. Erturk et al., in a study of 171 Turkish dry and wet skull specimens, found a clinically relevant MCP in $35.67 \%$ of dry specimens. ${ }^{11}$ In this study, a clinically relevant MCP was found in 52\% of white individuals $(n=1648)$, while only being present in $30 \%$ of individuals identified as black $(\mathrm{n}=$ $1068)(\mathrm{p}<0.0001)$. Furthermore, white race was a significant predictor of MCP presence, with an OR of 2.29 when compared with skulls of individuals categorized as black ( $\mathrm{p}<0.01)$. White race was also noted to be significantly associated with greater MCP height This is consistent with previous morphometric observations of a larger and longer pituitary fossa among North American whites. ${ }^{5,8,13,41}$ Wang et al. suggested that increased thickness and height could provide increased resistance for parasellar adenoma extension, ${ }^{40}$ an important radiological parameter that guides expanded endonasal surgeries for pituitary tumors with parasellar extensions.

The presence of an ICB, a fibro-osseous bridge extending from the ACP to the PCP, increases the difficulty with which the clinoid space is accessed and increases the risk of carotid laceration, particularly in transcranial microsurgery. ${ }^{17}$ Keyes found an ICB in $6.13 \%$ of 4374 parasellar areas, whereas Inoue et al. ${ }^{17}$ identified the ossified bridge in only $2 \%$ of parasellar regions studied. Furthermore, Wang et al. showed an incidence of $5.78 \%$ of osseous bridges between the ACP and the PCP. ${ }^{40}$ We identified an ICB in $4 \%$ of all parasellar regions examined $(n=5318)$ and found significantly greater incidence in conjunction with the presence of a clinically relevant MCP ( $p<0.0001)$, and more specifically with those of a CCR ( $p<0.0001)$. Preoperative knowledge of the presence of an ICB can facilitate surgical planning and risk counseling.

The vast majority of all MCPs, regardless of classification, were located within the anterior one-third of the LBW, consistent with the findings presented by Wang et $\mathrm{al}^{40}$ In this study, the superior aspect of the base of the $\mathrm{MCP}$ was found $6.25 \mathrm{~mm}$ posteromedially from the posterior border of the OS, and $3.70 \mathrm{~mm}$ inferior to the OCE. Peris-Celda et al., in a study of 66 dry skulls and 38 wet specimens, described the orientation of the MCP as an axial obtuse angle of $116.25^{\circ}$ posterior to the midsagittal plane, and directed toward the ACP in the coronal plane. They measured the base of the MCP $2.9 \mathrm{~mm}$ lateral to the lateral aspect of the TS, $1 \mathrm{~mm}$ inferolateral to the caroticosellar point, and $4.75 \mathrm{~mm}$ inferior to the medial opticocarotid point.$^{31}$ Of note, Labib et al. described the position of the MCP inferior to the LTC, a bony ridge situated at the superomedial aspect of the carotid sulcus (Fig. 4). ${ }^{24}$

There are limitations to the conclusions drawn in this investigation. First, the analysis of the soft-tissue structures and ligamentous attachments was not included as a morphometric variation in this survey of dry skull specimens. Second, the initial preparation and preservation techniques can cause morphological incongruence between dry and fresh skull specimens. Finally, we were unable to acquire CT angiography (CTA) images of these same skull specimens for correlation of vascular and softtissue relationships, especially the position of the ICA (encirclement, displacement, or bone erosion) in relation to CCR formation. Despite these limitations, our analysis followed the methodology identified and validated in other technical studies, anatomical surveys, and selected intraoperative videos from senior authors.

\section{Clinical Implications and Middle Clinoidectomy}

A paucity of literature exists pertinent to neurosurgery in terms of the intracranial relationships of the upper sphenoid process, particularly the correlative anatomy of the OS, ICA, and MCP comparing endonasal and microsurgical approaches. ${ }^{12,31,40}$

With wider anatomic visualization of the subchiasmatic and parasellar regions and the ability to remove medial tumors with ICA transposition, the endoscopic endonasal approach for surgical targets within the ventral base 


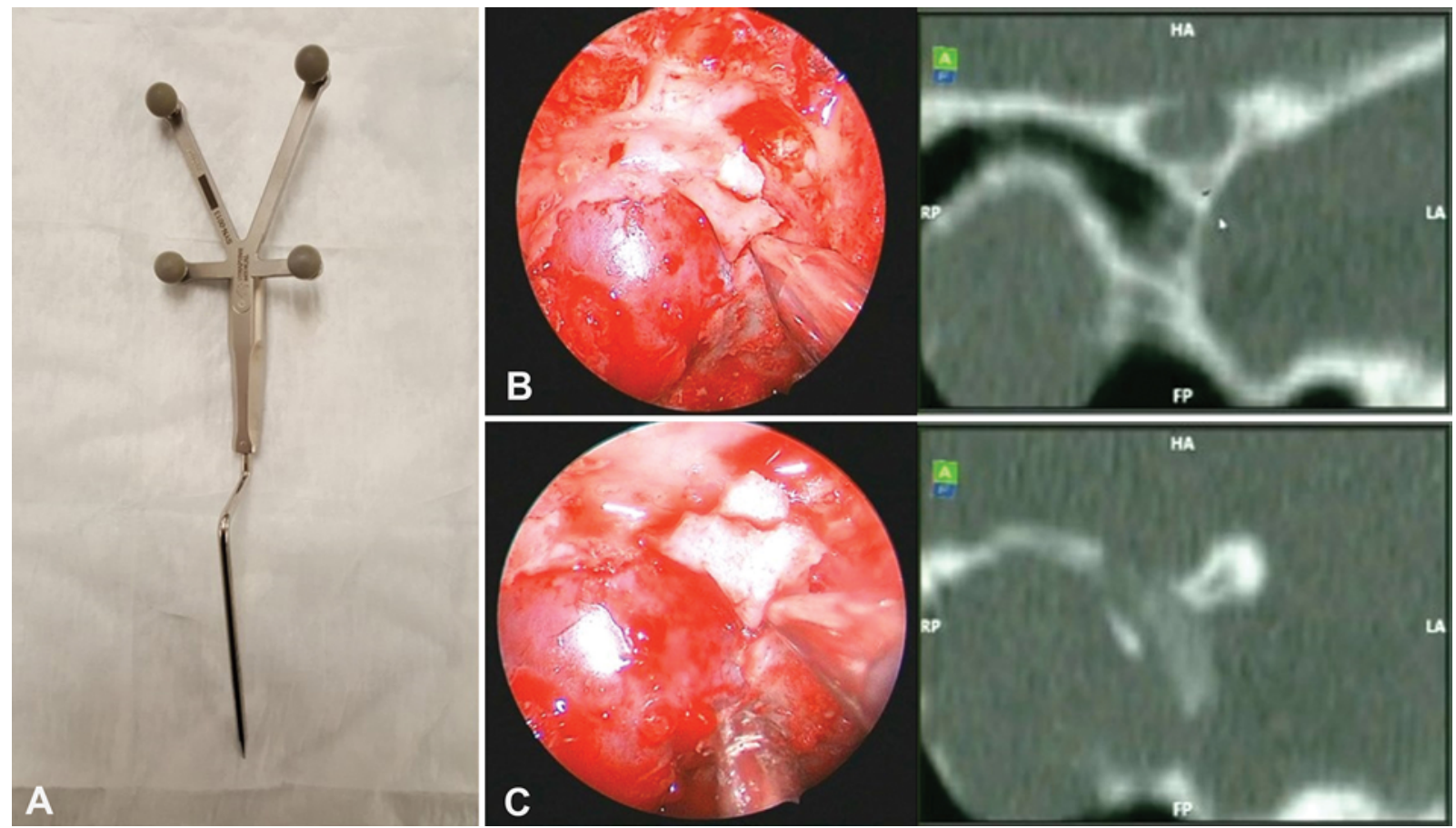

FIG. 6. The role of thin-cut CT imaging for intraoperative stereotaxy in parasellar approaches with MCPs in the endoscopic endonasal approach. A: Stryker intraoperative navigational probe with 4 radial fiducial markers. B and C: Endoscopic views of the left parasellar region via the EEA to access the MCP with concurrent visualization of the CTA bone windows, showing the CCR (B, right) and ICA (C, right) in stereotactic neuronavigation. Provided by Amin B Kassam, MD, Aurora Health Hospital, Milwaukee, Wisconsin. Figure is available in color online only.

of the skull has significantly revolutionized the access to and treatment of a number of neurological pathologies that were previously restricted by anatomical barriers. $.^{18,20,22} \mathrm{Al}-$ lowing access to cranial areas with significant neurovascular structures, the EEA has evolved in utility in tandem with advances in technology, but also with increasing anatomical understanding of the skull base. ${ }^{18,20}$ The MCP and the MOCR have been identified as 2 of the most important landmarks for EEA to the parasellar base. ${ }^{17}$ The lateral limits of EEA access have been identified as regions lateral to the paraclival and intercavernous ICA as well as the lower cranial nerves. ${ }^{7}$ Analogous to the ACP in pterional approaches, the MOCR is considered a crucial landmark to locate the opticocarotid cistern and provide control of the carotid artery and optic nerve; however, the role of the $\mathrm{MCP}$, especially in CCR, is understated. ${ }^{17}$ High-resolution imaging of the MOCR and its relationship to the carotid artery and the optic nerve are critical in creating the needed trajectory and in isolating the critical structures. In addition, they can represent anomalies such as ossified rings that can encase the carotid artery, or anomalous carotid positions such as dehiscence and significant vascular angulations, which put the carotid artery at risk to isolate during these procedures.

Significant challenges and limitations to the EEA remain, particularly in the identification of anatomical structures by using unfamiliar landmarks, ${ }^{38,40}$ as well as the development of surgical tools that can be accommodated in regions of limited space and access around critical neurovascular structures. ${ }^{6}$ The safety of the EEA can be enhanced via an intraoperative navigational probe coreg- istered with CT imaging - for example, Synaptive Medical technology (Fig. 6). We use a coarse hybrid 3- to 4-mm diamond drill bit exclusively to eggshell the bone, and then a right-angle blunt dissector to remove that portion of the bone (Stryker Corp.).

A safe technique for removal of the MCP is depicted in Fig. 7, and illustrative intraoperative videos are also provided from selected cases of the senior surgeon-authors (Videos 1 and 2). Middle clinoidectomy is a rare but complex operative technique in both regional EEA and microsurgical procedures, which requires specialized surgical tools and angles of operation to control force vectors and avoid inadvertent carotid artery injury (Fig. 8). ${ }^{1,2,4,12,30}$ To reduce the risk of ICA injury and allow surgical repair, visualization is integral: transcranial approaches should include an intradural component during the clinoidectomy for adequate exposure and visualization of relevant landmarks, whereas the EEA should include wide drilling around the OCR to delineate the medial and lateral boundaries of the ICA. ${ }^{4,28,29}$ Surgical complications also include CSF leakage, which can be managed effectively and safely with a vascularized reconstruction.

\section{Conclusions}

Our study elucidates the variation patterns of the MCP and demonstrates its operative significance in complication avoidance and management. An understanding of parasellar anatomy associated with racial and sexual variation allows for anticipation of potential intraoperative problems with adequate bone removal, and thus is integral 

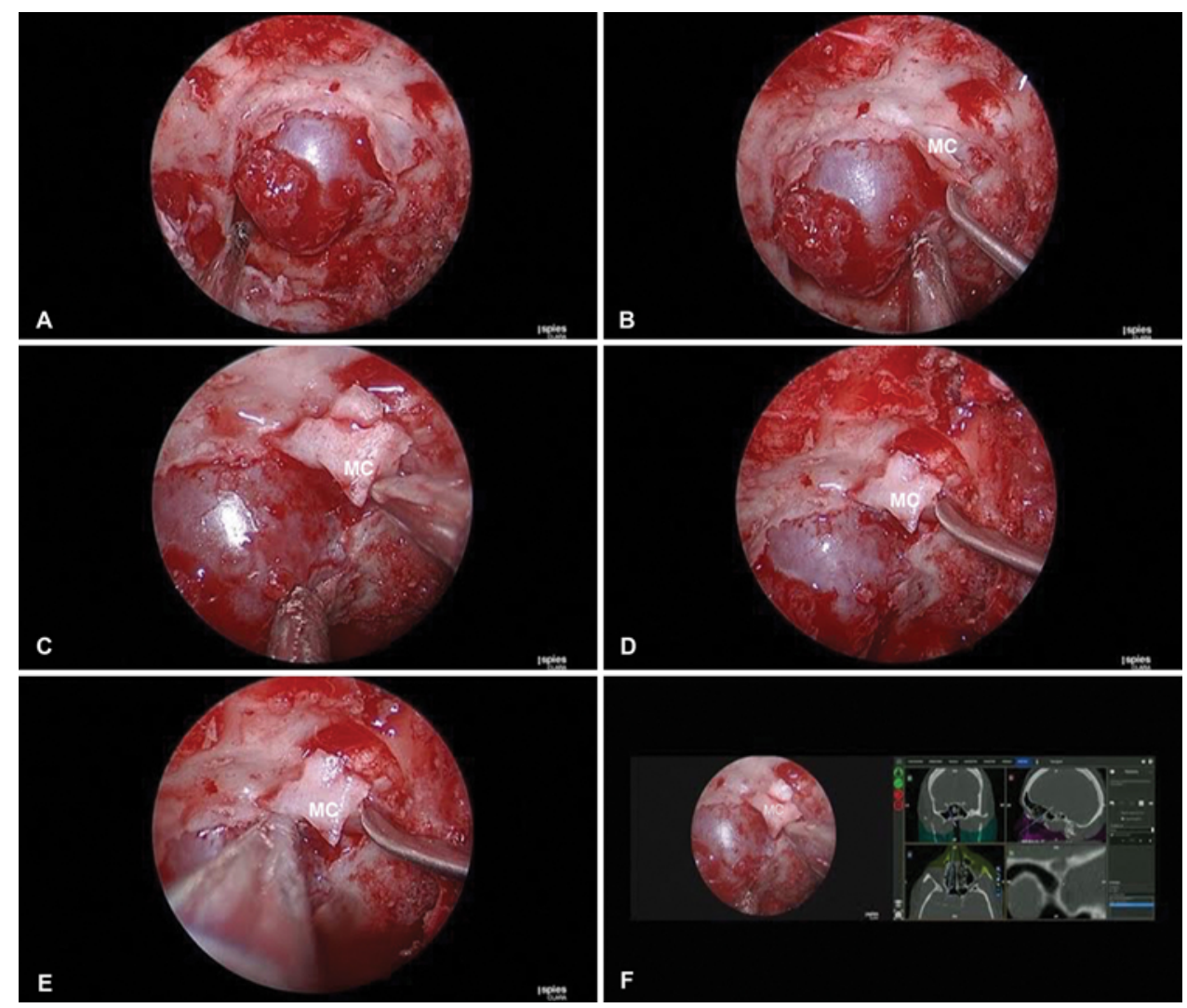

FIG. 7. Safe surgical technique for removal of MCP in the EEA. Endoscopic view of the sellar-parasellar region. A: Sellar floor. B-E: Close-up endonasal view of sequential steps of MCP removal, starting from microdissection separating the dura mater, drilling at the epicenter of MOCR/MCP, and eventually dissecting the bone overlying the transition point of clinoid and supraclinoid ICA, at the lateral extent of the TS, inferior to the distal cisternal segment of the optic nerve. F: The inset image of corresponding intraoperatively navigated CTA bone windows shows relevant anatomical landmarks. Provided by Amin B Kassam, MD, Aurora Health Hospital, Milwaukee, Wisconsin. MC = middle clinoid. Figure is available in color online only.
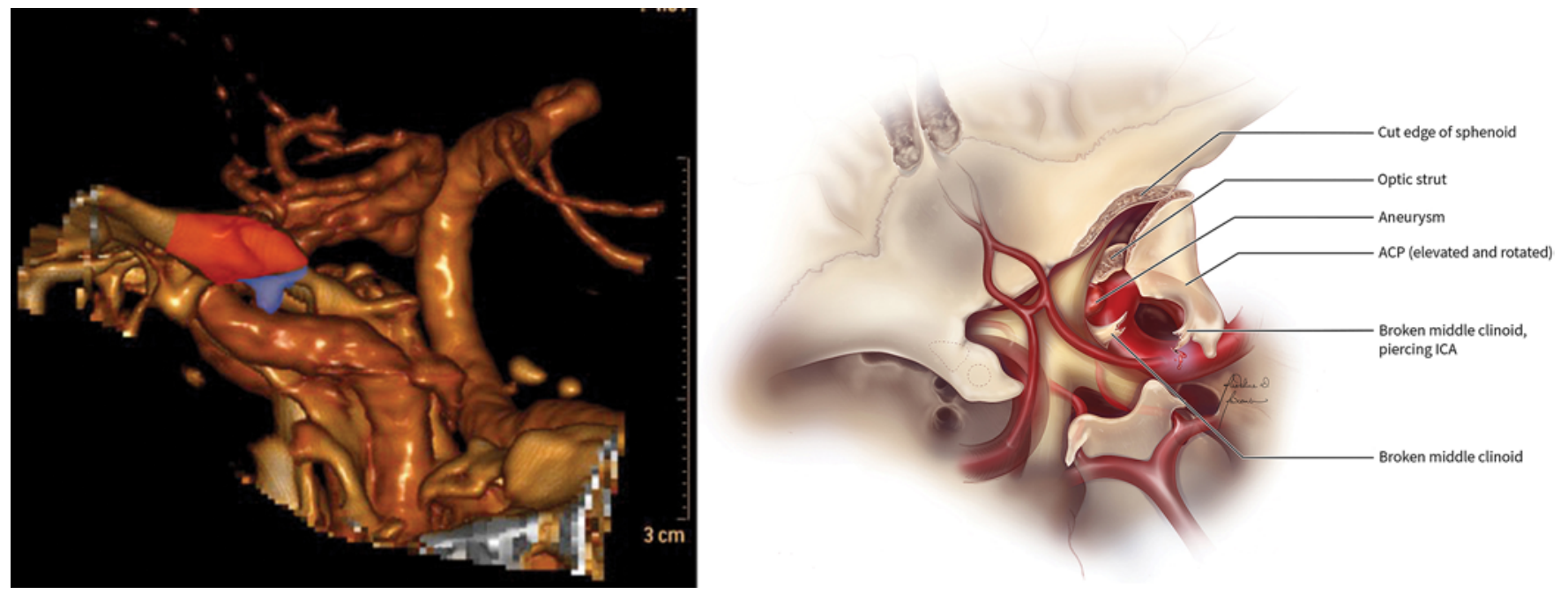

FIG. 8. Presence of a CCR can increase the risk of ICA injury during the surgical approach to the parasellar region. Left: A CTA image showing the ACP (red), MCP (blue), and PCP (natural coloring) in relation to the clinoid segment of the ICA. Right: Illustration of a fractured right MCP during microsurgical dissection, which can cause potentially fatal iatrogenic injury of the parasellar right ICA. Right panel copyright Madeline D. Newcomb. Published with permission. Figure is available in color online only. 
to surgical planning and preoperative patient counseling. This article highlights the relevant morphometric and radiological anatomy in reference to both the transcranial microsurgical approach and the EEA to the central skull base.

\section{Acknowledgments}

Special thanks to David Ponga, 3D CT technologist at the Aurora Neuroscience Innovation Institute, who assisted in acquiring representative CT images for interpretation of anatomical landmarks discussed in this manuscript.

\section{References}

1. Aggarwal B, Gupta M, Kumar H: Ossified caroticoclinoid ligament of sphenoid bone. Bombay Hosp J 53:743-746, 2011

2. Alleyne CH, Coscarella E, Spetzler RF, Walker MT, Patel AC, Wallace RC: Microsurgical anatomy of the clinoidal segment of the internal carotid artery, carotid cave, and paraclinoid space. Barrow Q 18:8-10, 2002

3. Azeredo RA, Liberti EA, Watanabe IS: [Anatomical variations of the clinoid process of the human sphenoid bone.] Arq Cent Estud Curso Odontol 25-26:9-11, 1988 (Portuguese)

4. Batjer HH, Welch BG: Respecting the clinoid: an application of preoperative computed tomography angiography. World Neurosurg 83:1022-1023, 2015

5. Burrows H, Cave AJE, Parbury K: A radiographical comparison of the pituitary fossa in male and female whites and negroes. Br J Radiol 16:87-89, 1943

6. Cavallo LM, de Divitiis O, Aydin S, Messina A, Esposito F, Iaconetta G, et al: Extended endoscopic endonasal transsphenoidal approach to the suprasellar area: anatomic considerations - part 1. Neurosurgery 61 (3 Suppl):24-34, 2007

7. Cutler AR, Mundi JS, Solomon N, Suh JD, Wang MB, Bergsneider M: Critical appraisal of extent of resection of clival lesions using the expanded endoscopic endonasal approach. J Neurol Surg B Skull Base 74:217-224, 2013

8. D'Aloisio D, Pangrazio-Kulbersh V: A comparative and correlational study of the cranial base in North American blacks. Am J Orthod Dentofacial Orthop 102:449-455, 1992

9. Das S, Suri R, Kapur V: Ossification of caroticoclinoid ligament and its clinical importance in skull-based surgery. Sao Paulo Med J 125:351-353, 2007

10. Dodo Y, Ishida H: Incidences of nonmetric cranial variants in several population samples from East Asia and North America. J Anthropol Soc Nippon 95:161-177, 1987

11. Erturk M, Kayalioglu G, Govsa F: Anatomy of the clinoidal region with special emphasis on the caroticoclinoid foramen and interclinoid osseous bridge in a recent Turkish population. Neurosurg Rev 27:22-26, 2004

12. Fernandez-Miranda JC, Tormenti M, Latorre F, Gardner P, Snyderman C: Endoscopic endonasal middle clinoidectomy: anatomic, radiological, and technical note. Neurosurgery 71 (2 Suppl Operative):ons233-ons239, 2012

13. Francis CC: Growth of the human pituitary fossa. Hum Biol 20:1-20, 1948

14. Freire AR, Rossi AC, Prado FB, Groppo FC, Caria PHF, Botacin PR, et al: Caroticoclinoid foramen in human skulls: incidence, morphometry and its clinical implications. Int J Morphol 29:427-431, 2011

15. Gupta N, Ray B, Ghosh S: A study on anterior clinoid process and optic strut with emphasis on variations of caroticoclinoid foramen. Nepal Med Coll J 7:141-144, 2005

16. Gurun R, Magden O, Ertem A: Foramen coticoclinoideym. Cerrahpasa Tip Derg 25:685-691, 1994
17. Inoue $\mathrm{T}$, Rhoton AL Jr, Theele $\mathrm{D}$, Barry ME: Surgical approaches to the cavernous sinus: a microsurgical study. Neurosurgery 26:903-932, 1990

18. Kassam A, Snyderman CH, Mintz A, Gardner P, Carrau RL: Expanded endonasal approach: the rostrocaudal axis. Part I. Crista galli to the sella turcica. Neurosurg Focus 19(1):E3, 2005

19. Kassam A, Snyderman CH, Mintz A, Gardner P, Carrau RL: Expanded endonasal approach: the rostrocaudal axis. Part II. Posterior clinoids to the foramen magnum. Neurosurg Focus 19(1):E4, 2005

20. Kassam AB, Gardner P, Snyderman C, Mintz A, Carrau R: Expanded endonasal approach: fully endoscopic, completely transnasal approach to the middle third of the clivus, petrous bone, middle cranial fossa, and infratemporal fossa. Neurosurg Focus 19(1):E6, 2005

21. Kassam AB, Gardner PA, Snyderman CH, Carrau RL, Mintz AH, Prevedello DM: Expanded endonasal approach, a fully endoscopic transnasal approach for the resection of midline suprasellar craniopharyngiomas: a new classification based on the infundibulum. J Neurosurg 108:715-728, 2008

22. Kassam AB, Prevedello DM, Carrau RL, Snyderman CH, Gardner P, Osawa S, et al: The front door to Meckel's cave: an anteromedial corridor via expanded endoscopic endonasal approach - technical considerations and clinical series. Neurosurgery 64 (3 Suppl):ons71-ons83, 2009

23. Keyes JL: Observations on four thousand optic foramina in human skulls of known origin. Arch Ophthalmol 13:538568,1935

24. Labib MA, Prevedello DM, Fernandez-Miranda JC, Sivakanthan S, Benet A, Morera V, et al: The medial opticocarotid recess: an anatomic study of an endoscopic "key landmark" for the ventral cranial base. Neurosurgery 72 (1 Suppl Operative):66-76, 2013

25. Lee HY, Chung IH, Choi BY, Lee KS: Anterior clinoid process and optic strut in Koreans. Yonsei Med J 38:151-154, 1997

26. Maxia C: Sul significato morfologico della frequenza dei processi clinoidei e della fusione nel cranio umano. Rass Med Sarda 1:1-7, 1950

27. Mensforth RP, Latimer BM: Hamann-Todd Collection aging studies: osteoporosis fracture syndrome. Am J Phys Anthropol 80:461-479, 1989

28. Ota N, Tanikawa R, Miyazaki T, Miyata S, Oda J, Noda K, et al: Surgical microanatomy of the anterior clinoid process for paraclinoid aneurysm surgery and efficient modification of extradural anterior clinoidectomy. World Neurosurg 83:635-643, 2015

29. Ota T, Mizutani T: Microscopic anterior clinoidectomy with micro-rongeurs for a superior projecting paraclinoid internal carotid artery aneurysm: a technical note. Br J Neurosurg 27:540-542, 2013

30. Ozdoğmuş O, Saka E, Tulay C, Gürdal E, Uzün I, Çavdar S: The anatomy of the carotico-clinoid foramen and its relation with the internal carotid artery. Surg Radiol Anat 25:241246, 2003

31. Peris-Celda M, Kucukyuruk B, Monroy-Sosa A, Funaki T, Valentine R, Rhoton AL Jr: The recesses of the sellar wall of the sphenoid sinus and their intracranial relationships. Neurosurgery 73 (2 Suppl Operative):ons117-ons131, 2013

32. Ray B, Gupta N: Caroticoclinoid foramen with interclinoid osseous bar. Clin Anat 19:732-733, 2006

33. Reisch R, Vutskits L, Filippi R, Patonay L, Fries G, Perneczky A: Topographic microsurgical anatomy of the paraclinoid carotid artery. Neurosurg $\operatorname{Rev} 25: 177-183,2002$

34. Renn WH, Rhoton AL Jr: Microsurgical anatomy of the sellar region. J Neurosurg 43:288-298, 1975

35. Rhoton AL Jr: The supratentorial arteries. Neurosurgery 51 (4 Suppl):S53-S120, 2002 
36. Rhoton AL Jr, Hardy DG, Chambers SM: Microsurgical anatomy and dissection of the sphenoid bone, cavernous sinus and sellar region. Surg Neurol 12:63-104, 1979

37. Shrout PE, Fleiss JL: Intraclass correlations: uses in assessing rater reliability. Psychol Bull 86:420-428, 1979

38. Snyderman CH, Pant H, Carrau RL, Prevedello D, Gardner P, Kassam AB: What are the limits of endoscopic sinus surgery?: the expanded endonasal approach to the skull base. Keio J Med 58:152-160, 2009

39. Srisopark SS: Ossification of some normal ligaments of the human skull which produce new structures: the pterygospinous and pterygoalar bars and foramina, and the caroticoclinoid foramen. J Dent Assoc Thai 24:213-224, 1974

40. Wang J, Wang R, Lu Y, Yao Y, Qi S: Anatomical analysis on the lateral bone window of the sella turcica: a study on 530 adult dry skull base specimens. Int J Med Sci 11:134-141, 2014

41. Zuckerman S: Age changes in the basicranial axis of the human skull. Am J Phys Anthropol 13:521-539, 1955

\section{Disclosures}

Dr. Kassam reports consulting relationships with Synaptive Medical and KLS Martin Group, and serves on an advisory board for Medtronic.

\section{Author Contributions}

Conception and design: Sharma, Rieth, Manjila, Yapicilar. Acquisition of data: Sharma, Rieth, Williams, Ota, Chakravarthi, Manjila, Kassam. Analysis and interpretation of data: Sharma, Rieth, Tanenbaum, Williams, Chakravarthi, Manjila, Kassam. Drafting the article: Sharma, Rieth, Manjila. Critically revising the article: Sharma, Tanenbaum, Manjila, Kassam, Yapicilar. Reviewed submitted version of manuscript: Sharma, Rieth, Manjila. Approved the final version of the manuscript on behalf of all authors: Sharma. Statistical analysis: Sharma, Tanenbaum. Study supervision: Manjila, Yapicilar.

\section{Supplemental Information \\ Videos}

Video 1. https://vimeo.com/210292868.

Video 2. https://vimeo.com/210294273.

\section{Previous Presentations}

Portions of this work were presented in abstract form at the AANS Annual Meeting in Chicago, IL, on April 30, 2016.

\section{Correspondence}

Akshay Sharma, Case Western Reserve University School of Medicine, 10900 Adelbert Rd., Cleveland, OH 44106. email: akshay.sharma@case.edu. 